NASA TECHNICAL NOTE
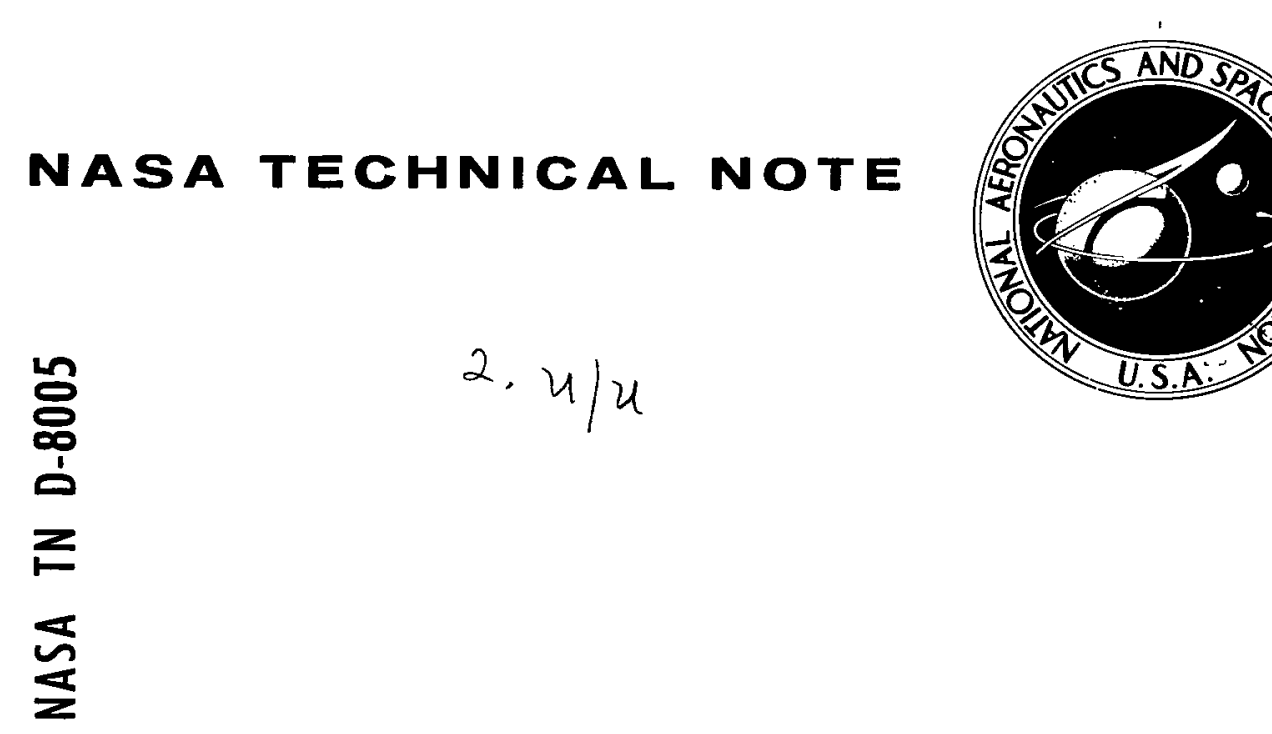

$\%$ NASA $/ \mathrm{TN} / \mathrm{D}-8005$ N COPY: RET TECHNICAL

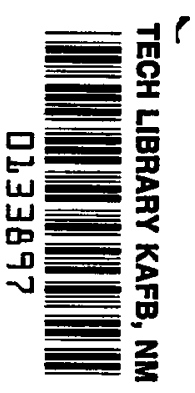

$x$.

EMISSION SPECTROMETRIC

ARCING PROCEDURE WITH

MINIMAL EFFECT OF

CHEMICAL FORM OF SAMPLE

William A. Gordon

Lewis Research Center

Cleveland, Obio 44135

$3^{\circ}$

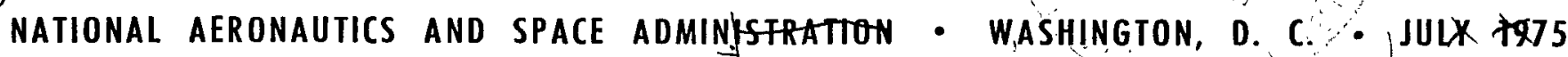



1. Report No.
N A SA TN D-8005
4. Title and Subtitle
EMISSION SPECTROMETRIC ARCING PROCEDURE WITH MINIMAL EFFECT OF CHEMICAL FORM OF SAMPLE
2. Government Accession No.

7. Author(s)

William A. Gordon

9. Performing Organization Name and Address

Lewis Research Center

National Aeronautics and Space Administration

Cleveland, Ohio 44135

12. Sponsoring Agency Name and Address

National Aeronautics and Space Administration

Washington, D.C. 20546

15. Supplementary Notes

1

3. Recipient's Catalog No.

5. Report Date

July 1975

6. Performing Organization Code

8. Performing Organization Report No. E-8284

10. Work Unit No. 505-01

11. Contract or Grant No.

13. Type of Report and Period Covered Technical Note

14. Sponsoring Agency Code

16. Abstract

This report describes a study of matrix effects related to the chemical form of materials analyzed. The study was made by using an arc in argon buffered with silver chloride. The effect of chemical form was minimal for a variety of metals, oxides, and carbides representing the most refractory compounds and thermally stable metal-containing molecules. Among these, only four of the most refractory materials known showed significant emission depressions due to incomplete volatilization in the arc system. These results are discussed in terms of vapor pressures of the solid materials placed on the anodes and dissociation reactions of the molecules in the gaseous environment.

17. Key Words (Suggested by Author(s))

Emission spectroscopy

Sample chemical form

Anode studies

Matrix effects

19. Security Classif. (of this report) Unclassified
18. Distribution Statement

Unclassified - unlimited

STAR Category 25 (rev.)

\footnotetext{
*For sale by the National Technical Information Service, Springfield, Virginia 22151
} 


\title{
EMISSION SPECTROMETRIC ARCING PROCEDURE WITH MINIMAL EFFECT OF CHEMICAL FORM OF SAMPLE
}

by William A. Gordon

Lewis Research Center

\author{
SUMMARY
}

This report describes a study of matrix effects related to the chemical form of materials analyzed by emission spectroscopy. The study was made by using a directcurrent arc in an argon atmosphere buffered with silver chloride. In a previous report these conditions were reported to be relatively free from matrix effects related to excitation of free atoms in the arc column. In the work of this report matrix effects related to sample chemistry were investigated by analyzing refractory compounds of high thermal stability placed directly on the anodes of the arc system. The analyses were done by using calibration standards of metals in halide acid solutions. The materials tested were aluminum ( $\mathrm{Al})$, aluminum oxide $\left(\mathrm{Al}_{2} \mathrm{O}_{3}\right)$, tungsten $(\mathrm{W})$, tungsten trioxide $\left(\mathrm{WO}_{3}\right)$, tungsten carbide $(\mathrm{WC})$, tantalum $(\mathrm{Ta})$, tantalum pentoxide $\left(\mathrm{Ta}_{2} \mathrm{O}_{5}\right)$, tantalum car bide ( $\mathrm{TaC})$, molybdenum (Mo), zirconium dioxide $\left(\mathrm{ZrO}_{2}\right)$, zirconium carbide $(\mathrm{ZrC})$, silicon carbide $(\mathrm{SiC})$, silicon dioxide $\left(\mathrm{SiO}_{2}\right)$, thorium dioxide $\left(\mathrm{ThO}_{2}\right)$, titanium carbide $(\mathrm{TiC})$, titanium dioxide $\left(\mathrm{TiO}_{2}\right)$, niobium $(\mathrm{Nb})$, niobium carbide $(\mathrm{NbC})$, and hafnium car bide $(\mathrm{HfC})$. In these experiments the quantity of sample recovered was generally within 10 percent of the amount present, and the analytical results were virtually independent of the chemical form of the material on the anode. Only four of the most refractory materials known gave significantly low analysis results. These were W, WC, TaC, and $\mathrm{NbC}$. Because these are the most refractory materials known, it was concluded that all other compounds will be quantitatively vaporized in the arc system. The minimal effects of sample chemistry of this arc system derive from five experimental features. First, the sample weighs only a few micrograms. Second, the sample is deposited as a thin film and experiences the maximum temperature of the anode spot. Third, the anode material is carbon, rather than graphite. Fourth, the discharge is conducted in an argon atmosphere, which minimizes the oxygen and carbon available in the gas phase and thereby minimizes formation of stable metal oxides and metal carbides. Fifth, the gaseous environment is at a high temperature. The experimental results are discussed in terms of vapor pressures of the solid materials on the anodes and the dissociation reactions in the arc environment. The relative freedom from effects of sample form greatly simplifies the application of this procedure in the analysis of acid-resistant materials because acid dissolution is not required. 


\section{INTRODUCTION}

One of the more troublesome characteristics of emission spectrochemical analysis is the dependence of emission intensity on the chemical form of the analytical specimen. Control of this effect involves critical and costly preparation of standards and samples to ensure that the chemical forms of the major and minor constituents in the sample are as nearly the same as those of the calibration standards as is possible. Inadequate control of this effect is possibly the primary cause of wide interlaboratory variability in analytical results. To avoid the practical problems encountered in converting samples to standard chemical forms and to improve analytical accuracy, excitation systems are needed in which the effects of sample chemistry are minimal. The purposes of this work, therefore, were to define quantitatively the effects of chemical forms of samples being analyzed by using the arc system described in reference 1 and to provide a more quantitative understanding of the processes in the arc relating to this problem. The arc system described in reference 1 is a silver chloride $(\mathrm{AgCl})$ - argon arc.

The primary processes occurring in the arc during spectrochemical analysis include the thermophysical reactions leading to the formation of free atoms and the subsequent excitation of atomic emission. These processes are discussed in detail for arcs in air in reference 2. The characteristics of the $\mathrm{AgCl}$-argon arc system with respect to excitation effects are described in reference 1 . The work of this report was primarily concerned only with those processes related to conversion of solid samples to free atoms in the arc column.

The formation of free atoms in the arc column is dependent on the vapor pressures of the solid constituents placed on the electrode and on the dissociation of molecules in the hot arc gas. The influence of these factors has been studied for arcs in air by examining the high-temperature reactions on graphite electrodes (refs. 3 to 5 ) and the thermochemical equilibria of stable monoxides (refs. 6 and 7). Although these studies have provided a better understanding of complex arc mechanisms, the practical problem of matrix effects caused by variable chemical forms is still controlled by closely matching the chemistry of standards and samples.

In the $\mathrm{AgCl}$-argon arcing procedure described previously (ref. 1), excitation conditions in the gas phase were effectively buffered by flooding the arc column with $\mathrm{AgCl}$. In this method the original chemical forms of the samples were destroyed by dissolution in acids. Small amounts of the acid solutions were dried on carbon anodes, and the chemical forms of the resulting residues were relatively invariable for both standards and samples. As a result of excitation buffering with $\mathrm{AgCl}$ and because the original sample chemistry was changed through acid dissolution, the calibration curves were applicable to a wide range of elemental compositions.

This residue arc procedure has been applied to the quantitative analysis of a wide variety of sample forms and compositions which are easily dissolved. However, 
because of limitations imposed by sample preparation, the procedure is of more limited use for materials which are not easily dissolved. Examples of samples which are rarely soluble in acids include products derived from superalloy oxidation research, particles filtered from the air, and other nondescript residues commonly received in a typical spectrochemical laboratory. Such materials often require more than 90 percent of the total analysis time for preparation of their solutions.

The data presented in this report establish that analysis results obtained by using the procedure specified are virtually independent of the chemical form of the materials placed on the anodes. Therefore, analysis of refractory oxides and other acid insoluble materials can be performed by using calibration curves prepared from metals in acid solution. With minimal effects of sample chemistry this procedure is now directly applicable to both liquid and solid samples.

\section{PROCEDURE}

To test the effects of sample form on emission intensities, water dispersions of selected compounds were prepared and pipetted onto carbon electrodes. The necessity of using carbon rather than graphite anodes is discussed in reference 1 . These electrodes, also containing 4 milligrams of $\mathrm{AgC1}$, were arced for 15 seconds in an argon atmosphere. The procedural details, including those relating to electrode configuration, arc chamber, and arcing program, are the same as those reported in reference 1. Therefore, in this section only those details which relate specifically to this work or are considered of special importance are described.

The time-integrated intensities of metal elements obtained with the solid compounds were compared with the intensities obtained with the same quantity of metal in acid solution. Differences observed were classified as being within or outside the experimental error. On the basis of these data, the effects of sample chemistry in this arcing system were generalized in terms of vapor pressures and molecular stability.

\section{Materials Tested}

The compounds and metals tested were selected because they represent intermediates and extremes with respect to low vapor pressure and high molecular stability. These materials included aluminum (Al), aluminum oxide $\left(\mathrm{Al}_{2} \mathrm{O}_{3}\right)$, tungsten (W), tungsten trioxide $\left(\mathrm{WO}_{3}\right)$, tungsten carbide (WC), tantalum (Ta), tantalum pentoxide $\left(\mathrm{Ta}_{2} \mathrm{O}_{5}\right)$, tantalum carbide $(\mathrm{TaC})$, molybdenum $(\mathrm{Mo})$, zirconium dioxide $\left(\mathrm{ZrO}_{2}\right)$, zirconium car bide $(\mathrm{ZrC})$, silicon carbide $(\mathrm{SiC})$, silicon dioxide $\left(\mathrm{SiO}_{2}\right)$, thorium dioxide $\left(\mathrm{ThO}_{2}\right)$, 
titanium carbide $(\mathrm{TiC})$, titanium dioxide $\left(\mathrm{TiO}_{2}\right)$, niobium $(\mathrm{Nb})$, niobium carbide $(\mathrm{NbC})$, and hafnium carbide ( $\mathrm{HfC})$. All of these materials had greater than 99 -percent purity .

\section{Preparation of Dispersions}

All of the materials tested were obtained as powders, usually -325 mesh. The brittle compounds were further hand ground in a boron carbide mortar with ethanol to form a paste. The ethanol was evaporated, and the ground material was weighed into glass vials. Water was added to obtain a concentration of about 0.5 milligram per cubic centimeter. The mixture was either shaken by hand or placed in an ultrasonic water bath to break up agglomerates and to achieve a good dispersion of particles.

\section{Accuracy of Pipetting Procedure}

A precision 0.010-cubic-centimeter pipette was used to transfer the dispersed material onto carbon electrodes. The pipette accuracy for true solutions was better than 1 percent. However, because of particle settling during the pipetting operation, the accuracy of transferring solid dispersions in this way was determined independently. This was done by recovery experiments in which the dispersed material was shaken by hand and a 0.010-cubic-centimeter aliquot was immediately transferred to a weighing pan. Five 0.010-cubic-centimeter aliquots were combined on the weighing pan, dried at $150^{\circ} \mathrm{C}$, and weighed with a microbalance having a direct readability of 0.1 microgram. Typical weights were between $25 \pm 2$ and $45 \pm 2$ micrograms. All of these pipetting operations closely simulated the experimental procedure for transferring the dispersions and drying them on the carbon anodes. With the gravimetric procedure as a check, relative errors in the pipetting operations were kept within about 5 to 10 percent.

\section{Addition of Dispersions to Carbon Anodes}

Four milligrams of $\mathrm{AgCl}$ was formed on each electrode before adding the dispersion sample. The $\mathrm{AgCl}$ was formed on the electrode by first adding 0.010 cubic centimeter of 2.8 nor mal silver nitrate $\left(\mathrm{AgNO}_{3}\right)$ solution and then adding 0.010 cubic centimeter of 6 normal hydrochloric acid. The dispersed material was added to the carbon anodes at $150^{\circ} \mathrm{C}$ by using a 0.010 -cubic-centimeter pipette. The liquid was immediately adsorbed as a thin layer within a few millimeters of the tip of the porous carbon electrode and dried within about 5 seconds. The determination of the metal constituent of each compound in aqueous dispersion and its respective acid solution was repeated five times. 


\section{Arcing Procedure}

The $\mathrm{AgCl}$ residue and the particulate sample were vaporized from the electrodes over a 15-second period in an argon atmosphere. The arc current was 10 amperes at the beginning of the arcing cycle and 30 amperes at the end of the arcing cycle. The integrated emission intensities were recorded for the entire 15 -second arcing cycle.

\section{Optical Aperture and Arc Geometry}

In experiments involving measurement of atomic emission from arcs, the results and their interpretation are strongly dependent on the region of the arc column under observation. The arc is inhomogeneous both axially and in cross section with respect to electron, atom, and ion densities and with respect to temperature. Therefore, specification of the optical system linking the arc with the spectrometer is appropriate.

The grating was the limiting optical aperture of the spectrometer and external optical system. The vertical arc was focused on the grating-collimator in a spectrometer of 0.75 -meter focal length. The geometry of the object field bounded by the arc column is shown in figure 1. The field was formed by crossed cylindrical lenses external to the spectrometer. Emissions originating in this field and intercepting the gratingcollimator aperture were detected and recorded.

\section{Analysis of Solid Materials}

The analysis procedure for solid materials was substantially identical with that described in the section Arcing Procedure for performing tests of vaporization. The sample was finely ground in a mortar, weighed, and warmed in an acid mixture consisting of three parts 38 percent hydrochloric acid $(\mathrm{HCl})$ and one part 70 percent nitric acid $\left(\mathrm{HNO}_{3}\right)$. One cubic centimeter of the acid mixture was added per 10 milligrams of sample. This mixture was then diluted with water so that the resulting dispersion contained 1 milligram of sample per cubic centimeter of liquid. The dosing of dispersions and the arcing procedure were likewise the same as those described for acid solutions in reference 1. The acid concentration of the final solution was not critical. Whether the solid material went into solution, decomposed, or remained as an inert suspension was immaterial with this procedure. However, if the solid material is even partially decomposed by the acid, then quantitative verification of material transfer in the dosing operations cannot be done by using the gravimetric procedure described in the section Preparation of Dispersions because of uncertain stoichiometry. Therefore, practice 
with the gravimetric method using water dispersions is desirable to gain experience in processing dispersions.

The time integrated intensities were transformed to absolute amounts of metals by using known concentrations of metals in solutions (ref. 1). The reference standards were appropriate blends of single elements in solutions covering the desired ranges of the metals being analyzed.

\section{RESULTS AND DISCUSSION}

\section{Experiments on Effects of Chemical Form}

Table I is a summary of the results of the experiments on the effects of chemical form. The table lists the chemical forms of the materials applied directly to the carbon anode and also the acid media used for the calibration solution. The last column presents the relative difference between the amount of solid material added and the amount determined. This difference indicates the potential relative error in the analysis re:sults that might be caused by sample form. The only compounds that were not quantitatively recovered, as indicated by relative differences exceeding the experimental error of about 10 percent of the amount present, were $\mathrm{W}, \mathrm{WC}, \mathrm{NbC}$, and $\mathrm{TaC}$.

\section{Analysis of Typical Dispersion}

Table II is a summary of analysis results obtained on a sample of material that is insoluble in acids. This material is a chrome refractory ore designated by the National Bureau of Standards as the standard NBS-103. This material typifies a class of materials which are insoluble in mineral acids and which usually require alkali fusions preliminary to dissolution. The most effective method for dissolution of this standard is fusion in sodium peroxide. The accuracy of results shown in table II is typical of the analysis of aqueous dispersions and is acceptable for many specimens of miscellaneous residues which are submitted to a spectrochemical laboratory.

When samples containing compounds other than those investigated in this work are analyzed, the effect of sample chemistry can be estimated by generalizing the results in table I. These generalizations were made on the basis of thermophysical properties of the compounds, namely, vapor pressures and dissociation constants, as discussed in the next section. 
Model of Vaporization and Atomization in Silver Chloride - Argon Arc

Figure 2 illustrates schematically the processes that occur in the AgCl-argon arc when solid materials on the anode are converted to free atoms in the arc column. These processes ultimately produce the light emission which is the basis of quantitative measurements. The three processes illustrated in figure 2 that relate to matrix effects of sample chemistry are volatilization, molecular dissociation, and emission excitation. The figure shows possible solid-state reactions that involve formation and dissociation of carbides, oxides, or chlorides because these are the predominant reactive species in this arc system. The vapor pressure and thus the rate of volatilization are dependent on the specific compounds either deposited initially or formed on the anode by chemical reaction.

Vaporization. - The data in table I indicate the importance of vapor pressure inasmuch as only $\mathrm{TaC}, \mathrm{NbC}, \mathrm{W}$, and $\mathrm{WC}$ were apparently incompletely vaporized. This suggests that the formation of chlorides producing volatile $\mathrm{W}$ compounds, for example, is not a primary reaction with these arcing conditions. The correlation between the vapor pressures and the differences in emission intensities for the metals as solids and in acid solutions is shown in figure 3 . In this plot the vapor pressures of the metal over the metal carbide with excess carbon and the vapor pressures of the metal over the metal are plotted for data obtained at $3000 \mathrm{~K}$ (refs. 8 to 10). This temperature was ar bitrarily selected because it was approximately the highest temperature at which data were available for the materials of interest and was also the approximate temperature prevailing on the anode surface. The plot clearly shows the correlation with vapor pressures. At a pressure of about $10^{-7}$ atmosphere $\left(10^{-2} \mathrm{~Pa}\right)$ there is a rather abrupt decr.ease from 100 percent vaporization to about 20 percent or less. With this correlation it was possible to generalize the effect of vapor pressure on percent vaporization. On the basis of this figure we concluded that any material with vapor pressure greater than that of $\mathrm{HfC}$ will be quantitatively vaporized in this arc system. The only compounds known to have vapor pressures lower than $\mathrm{HfC}$ are those included in this study, namely, $\mathrm{NbC}, \mathrm{WC}, \mathrm{W}$, and TaC. In addition, we presume that compounds that decompose to these forms will also be incompletely vaporized. Therefore, the hundreds of other metals and compounds analyzable by this arc system will be quantitatively vaporized.

This model of vaporization as a function of vapor pressure does not suggest, however, that the total production of vapor necessarily occurs on the carbon anodes. There exists the possibility that materials entrained by the $\mathrm{AgC} 1$ vapor are carried from the anode up into the arc column and are vaporized there. This mechanis $m$ is reminiscent of the so-called carrier effect (ref. 11), which has not been satisfactorily explained. Atomization. - After the sample material is transported into the hot arc column, the thermal dissociation of molecular species is necessary to form the desired free 
atoms. Incomplete dissociation is a type of matrix effect which is related to sample chemistry. The experimental results of table I indicate that thorium monoxide (ThO), the most stable metal-containing molecule known, was quantitatively dissociated in the arc column. It was therefore concluded that analysis errors caused by incomplete dissociation probably were not significant in this arc. However, because ThO was neither directly observed nor shown to be present in the arc gas, an approximate model of molecular equilibrium was developed to determine if estimates from arc theory were consistent with experimental observations.

To estimate the percent error caused by dissociation equilibria in the arc column several assumptions were made. First, and most important, we assumed a steadystate condition in the arc during the vaporization process so that equilibrium constants for molecular dissociation could be used to estimate concentrations of molecules and atoms. Second, we assumed a condition of local thermal equilibrium with an effective temperature averaged over the arc volume from which the light emission was taken. These assumptions are generally considered valid within the limitations discussed in reference 2. The effective temperature was measured by using the 307.2- and 307.6nanometer atomic zinc lines, according to the two-line method of reference 2. The volume-averaged temperature for the $\mathrm{AgCl}$-argon arc was $6000 \mathrm{~K}$. Because of these assumptions and others detailed in reference 2 , the calculations of partial pressures are accurate only to within about a factor of 10. However, the estimates of the percentage of analysis error are accurate within a factor of approximately 2 to 3 .

Because metal monoxides are the most thermally stable metal-containing molecules and because oxygen molecules are about 95-percent dissociated at $6000 \mathrm{~K}$, the equilibrium expression of interest is

$$
\mathrm{M}(\mathrm{g})+\mathrm{O}(\mathrm{g})=\mathrm{MO}(\mathrm{g})
$$

where $M$ is the metal atom and $M O$ is the metal monoxide.

The formation of metal carbides and metal halides is less likely in this arc than the formation of monoxides because the carbides and halides generally have lower dissociation energies than the monoxides. In spite of the fact that carbon electrodes are used in this arc, a very minute amount of carbon is vaporized. From spectral line intensities the partial pressure of carbon is estimated to be about the same as the oxygen partial pressure that results from dissociation of metal oxides. Furthermore, the formation of the exceedingly stable carbon monoxide molecule $(\mathrm{CO})$ is also ignored in this analysis. Formation of $\mathrm{CO}$ would further reduce the availability of carbon and oxygen to combine with metal atoms. Thus, the predictions of analysis errors due to incomplete dissociation based on equation (1) represent upper error limits.

A ranking of the most stable monoxides, taken from reference 12 , is shown in table III. Of the monoxides listed, $\mathrm{ThO}, \mathrm{TaO}, \mathrm{SiO}, \mathrm{ZrO}$, and $\mathrm{NbO}$ were derivable 
reactants from the oxides included in our tests. Values of the equilibrium constant $K_{p}$ for these reactants at temperatures up to $6000 \mathrm{~K}$ are given in reference 13 . From equation (1) the expression relating partial pressures of the metal, oxygen, and the monoxide is

$$
\mathrm{K}_{\mathrm{p}}=\frac{\mathrm{P}_{\mathrm{MO}}}{\mathrm{P}_{\mathrm{M}^{\mathrm{P}} \mathrm{O}}}
$$

Since the reaction takes place in an argon atmosphere with an oxygen impurity of less than $10 \mathrm{ppm}$, the partial pressure of oxygen is primarily fixed by the decomposition of $\mathrm{MO}_{\mathrm{x}}$. For $\mathrm{x}=2$,

$$
{ }^{2 P_{M}}=\mathrm{P}_{\mathrm{O}}
$$

and

$$
\mathbf{P}_{\mathbf{T}}=\mathbf{P}_{\mathbf{M}}+\mathbf{P}_{\mathbf{M O}}
$$

where $\mathrm{P}_{\mathrm{T}}$ is the total partial pressure of metal-containing particles. Through substitution of equations (3) and (4) in equation (2) we obtain

$$
\mathrm{P}_{\mathrm{MO}}=2 \mathrm{~K}_{\mathrm{p}}\left(\mathrm{P}_{\mathrm{T}}-\mathrm{P}_{\mathrm{MO}}\right)^{2}
$$

From equation (5) we calculate the ratio of thorium metal to thorium monoxide as a function of the total pressure of metal-containing species $P_{T}$. This ratio is in terms of partial pressures and is approximately equal to the ratio of particles for heavy metals such as thorium. The estimate of this ratio requires an estimate of $P_{T}$. This estimate was made from the known quantity of metal oxide placed on the anode, the volume and temperature of the arc column, and the average lifetime of the vapor in the arc column. The lifetime is also referred to as the transit time. The details of this estimate are given in the appendix.

The value of $P_{T}$ estimated by this method was $5 \times 10^{-6}$ atmosphere $(0.5 \mathrm{~Pa})$. At this total pressure, application of equation (5) shows that the fraction of undissociated ThO is less than 1 part per 10000 of total metal. This represents a totally negligible analysis error. In fact, to produce a pressure ratio of 1 part ThO to 10 parts thorium (Th), the approximate experimental error of data in table I, requires a total partial pressure of about $10^{-2}$ atmosphere $\left(10^{-3} \mathrm{~Pa}\right)$. This is estimated to be several orders of magnitude higher than metal vapor pressures in the $\mathrm{AgCl}$-argon arc under the conditions 
used in this work. From this analysis we concluded that effects of sample chemistry due to incomplete thermal dissociation were negligible in our experiment.

This conclusion was recently confirmed in an unpublished study by Fred J. Kohl of this laboratory. Using a computer program for calculating complex chemical equilibrium compositions (ref. 14), he made a more complete thermodynamic analysis of the equilibrium species in this arc system, including silver, chlorine, argon, carbon, oxygen, and thorium. The results showed that the composition of thorium-containing species was about 99.9 percent Th at $6000 \mathrm{~K}$, and about 99.5 percent $\mathrm{Th}$ at $5000 \mathrm{~K}$. The calculations did not take into account the oxygen supply from other oxides which may be present in a real analytical specimen. However, inasmuch as the maximum amount of specimen usually vaporized is 30 micrograms, about six times the amount tested, this additional source of oxygen will also be negligible.

\section{CONCLUDING REMARKS}

In this report, conditions are described under which the integrated emission intensities of metal compounds are minimally dependent on the chemical for $\mathrm{m}$ of the samples. Among those materials tested only a few refractory materials, including $\mathrm{W}$, WC, $\mathrm{NbC}$, and $\mathrm{TaC}$, gave low emission intensities of the metal constituents compared with the same amount of metal in acid solution. Because these are the most refractory materials known, it was concluded that all other metals and compounds will be quantitatively removed from the anode. From experimental evidence, supported by thermochemical estimates, it was also concluded that effects of sample form related to incomplete molecular dissociation are also negligible. These desirable characteristics of the arc system derive from five experimental features. First, the sample weighs only a few micrograms and is vaporized to completion. Second, the sample is deposited as a thin layer and experiences the maximum temperature of the anode spot. Third, the anode material is carbon, rather than graphite. Fourth, the discharge is conducted in an argon atmosphere, which minimizes the oxygen and carbon available in the gas phase and thereby minimizes for mation of stable metal oxides and metal carbides. Fifth, the gaseous arc environment is at a high temperature. In addition, particles may be removed from the anode surface to the hot arc gas by entrainment by the silver chloride vapor; this possibility was not confirmed.

These properties of the arc system make it well suited for quantitative analysis of solid materials without prior dissolution. Furthermore, the quantitative calibrations for these analyses are conveniently made from single elements in acid solutions. The accuracy of analysis is presently limited to about 10 percent of the amount present with the primary source of error being the method of sampling very small volumes of dispersions which undergo sedimentation. In applications wherein this accuracy is acceptable, the analyses are uniquely simple. Sample preparation of even the most 
acid-resistant materials takes typically about 5 minutes, including time to pulverize the solids and prepare liquid dispersions, whereas some corrosion-resistant materials require hours of chemical processing to effect their dissolution.

Lewis Research Center,

National Aeronautics and Space Administration,

Cleveland, Ohio, April 25, 1975, 505-01. 


\section{APPENDIX - ESTIMATE OF METAL - METAL MONOXIDE RATIOS IN ARC COLUMN}

Partial pressures of metal and monoxide in the arc column cannot be calculated with precision because of lack of data for arcs in general and for the AgCl-argon arc in particular. Estimates can be made, however, from available data to within a factor of about 10 of the actual pressures.

Equation (1) represents a model of vapor concentration in the arc column; it is a greatly simplified model which assumes a homogeneous steady-state condition for processes that are highly dynamic:

$$
\mathrm{P}_{\mathrm{M}+\mathrm{MO}}=\mathrm{f} \frac{\tau}{\mathrm{t}} \frac{\mathrm{g}}{\mathrm{A}} \frac{\mathrm{RT}}{\mathrm{V}}
$$

where

f fraction of anode material entering discharge zone

$\tau \quad$ transit time of atoms and molecules in arc column, sec

$t \quad$ total vaporization time of material on anode, sec

$\mathrm{g} / \mathrm{A} \quad$ moles of material vaporized

R gas constant, $82.1\left(\mathrm{~cm}^{3}\right)(\mathrm{atm}) /($ mole $)(\mathrm{K})$

T gas temperature, $\mathrm{K}$

$\mathrm{V}$ volume of arc column, $\mathrm{cm}^{3}$

The number of moles of metal plus metal monoxide placed on the electrode is known, as is the total time of vaporization $t$. The time-averaged vaporization rate is calculated from measured emission intensities to be about one-half of the peak rate. The timeaveraged rates are used in this estimate. The emission rates were previously shown to be directly proportional to metal concentrations in the arc column (ref. 1).

The volume of the arc column $\mathrm{V}$ can be estimated from the diagram of figure 1 . With an interelectrode spacing of about 1 centimeter, the total arc volume is about 1 cubic centimeter.

The fraction of material actually entering this volume compared with the total amount that enters the larger volume of the surrounding chamber is $f$. Of all the parameters of equation (A1), the estimate of $f$ has the greatest error limit, perhaps as much as a factor of 10 . In this analysis we assume $\mathrm{f}=1$, that is, all the vaporized material enters the interelectrode volume. Therefore, the estimates of partial pressures and predicted analysis errors will be high. 
The mean lifetime of atoms in the arc volume is estimated to be about 5 milliseconds from experimental data for arcs in air (ref. 2). This estimate is probably within a factor of about 2 of the actual value.

The temperature $T$ was measured spectroscopically at $6000 \mathrm{~K}$, as discussed in the section Atomization. It should be understood that this temperature is a volumeaveraged one.

When the estimates just given are used in equation (A1), the partial pressure of thorium plus thorium monoxide is

$$
\begin{gathered}
\mathrm{P}_{\mathrm{Th}+\mathrm{ThO}} \simeq \frac{5 \times 10^{-3}}{10} \frac{5 \times 10^{-6}}{232}(82.1)\left(6 \times 10^{3}\right) \\
\mathrm{P}_{\mathrm{Th}+\mathrm{ThO}} \simeq 5 \times 10^{-6} \text { atm }(0.5 \mathrm{~Pa})
\end{gathered}
$$




\section{REF ERENCES}

1. Gordon, William A.; and Chapman, Gilbert B.: Quantitative Direct-Current Arc Analysis of Random Compositions of Microgram Re'sidues in Silver-Chloride Common Matrix. Spectrochimica Acta, vol. 25B, 1970, pp. 123-137.

2. Boumans, Paul W. J. M.: Theory of Spectrochemical Excitation. Plenum Press, 1966.

3. Nickel, H.: Considerations on the Reaction and Evaporation Mechanism of OxideContaining Graphite Electrodes During Arc Excitation. Presented at the XIIIth Colloquim Spectroscopicum Internationale, Ottawa, Canada, June 19-23, 1967.

4. Semenenko, K. A.; Tarasevich, N. I.; Vigant, G. T.; and Gul'ko, N. I. : Study of Processes in the Direct Current Arc During Spectrographic Determination of $\mathrm{Nb}$ and Ta. Zh. Anal. Khim., vol. 29, no. 6, June 1974, pp. 1113-1117.

5. Rautschke, Ruth; Amelung, Gerlinde; Dowe, C. ; and Nada, Nadra: Kinetic Thermochemical Reactions of Uranium Compounds in a Graphite Matrix and Its Influence on the Spectral Line Intensity. Z. Chem., vol. 13, no. 8, 1973, pp. 305-306.

6. Antic, E.; and Caro, P.: Influence des tampons spectroscopiques et de l'atmosphere gaseuse sur l'excitation du lanthane dans les plasmas d'arc e'lectrique. Spectrochimica Acta, vol. 27B, 1972, pp. 479-502.

7. Semenenko, K. A.; Gul'ko, N. I.; and Khramova, G. T.: Calculation of the Degrees of $\mathrm{TiO}$ and VO Dissociation and of Vanadium and Titanium Ionization in a Direct Current Arc Plasma. Khimiya, vol. 27, no. 4, 1972, pp. 481-483. (English Trans., UDC 543.42: 546.831).

8. Stearns, Carl A.; and Kohl, Fred J.: High-Temperature Mass SpectrometryVaporization of Group IVB Metal Carbides. NASA TN D-7613, 1974.

9. Hultgren, Ralph; Desai, Pramon D.; Hawkins, Donald T.; Gleiser, Molly; Kelley, Kenneth; and Wagman, Donald D.: Selected Values of the Thermodynamic Properties of the Elements. American Society for Metals, Metals Park, 1973.

10. Storms, Edmund K.: The Refractory Carbides. Academic Press, 1967.

11. Scribner, Bourdon F.; and Mullin, Harold R. : Carrier-Distillation Method of Spectrographic Analysis And Its Application to the Analysis of Uranium-Base Materials. J. Res. Nat. Bur. Std., vol. 37, Dec. 1946, pp. 379-389.

12. Drowart, J.; and Goldfinger, P.: Investigation of Inorganic Systems at High Temperature by Mass Spectrometry. Angewandte Chemie., vol. 6, no. 7, July 1967, pp. 581-596. 
13. Schick, Harold L., ed.: Thermodynamics of Certain Refractory Compounds. Vol. II, Thermody namic Tables, Bibliography and Property File, Sections VII, VIII, and IX. Academic Press, New York, 1966.

14. Gordon, Sanford; and McBride, Bonnie J.: Computer Program of Calculation of Complex Chemical Equilibrium Compositions, Rocket Performance, Incident and Reflected Shocks, and Chapman-Jouguet Detonations. NASA SP-273, 1971. 
TABLE I. - RESULTS OF EXPERIMENTS ON EFFECT

OF CHEMICAL FORM

\begin{tabular}{|c|c|c|c|c|}
\hline \multicolumn{2}{|c|}{ Element or compound } & \multicolumn{2}{|c|}{ Amount of metal, $\mu \mathrm{g}$} & \multirow{2}{*}{$\begin{array}{l}\text { Difference be- } \\
\text { tween amounts } \\
\text { added and de- } \\
\text { termined, } \\
\text { percent }\end{array}$} \\
\hline Calibration solution & $\begin{array}{l}\text { Analyzed } \\
\text { form }\end{array}$ & Added & Determined & \\
\hline \multirow[t]{2}{*}{$\mathrm{Al}$ in $\mathrm{HCl}$} & $\mathrm{Al}$ & 5.0 & 5.2 & +4 \\
\hline & $\mathrm{Al}_{2} \mathrm{O}_{3}$ & 5.0 & 4.9 & -2 \\
\hline \multirow[t]{3}{*}{$\mathrm{W}$ in $\mathrm{HF}$ and $\mathrm{HNO}_{3}$} & $W$ & 5.0 & 0.8 & $a_{-84}$ \\
\hline & $\mathrm{WO}_{3}$ & 5.0 & 5.1 & +2 \\
\hline & WC & 5.0 & 2.4 & $a_{-52}$ \\
\hline \multirow[t]{3}{*}{$\mathrm{Ta}$ in $\mathrm{HF}$ and $\mathrm{HNO}_{3}$} & $\mathrm{Ta}$ & 4.2 & 4.4 & +5 \\
\hline & $\mathrm{Ta}_{2} \mathrm{O}_{5}$ & 5.0 & 4.5 & -10 \\
\hline & $\mathrm{TaC}$ & 4.3 & .87 & a-87 \\
\hline Mo in $\mathrm{HF}$ and $\mathrm{HNO}_{3}$ & Mo & 5.0 & 5.0 & 0 \\
\hline \multirow[t]{2}{*}{$\mathrm{Zr}$ in $\mathrm{HF}$ and $\mathrm{HNO}_{3}$} & $\mathrm{ZrO}_{2}$ & 5.0 & 5.5 & +10 \\
\hline & $\mathrm{ZrC}$ & 5.0 & 5. 4 & +8 \\
\hline \multirow{2}{*}{$\mathrm{Si}$ in aqueous $\mathrm{K}_{2} \mathrm{SiO}_{3}$} & $\mathrm{SiC}$ & 5.0 & 5.3 & +6 \\
\hline & $\mathrm{SiO}_{2}$ & 5.0 & 5.4 & +8 \\
\hline $\mathrm{Th}$ in $\mathrm{HCl}$ and $\mathrm{HF}$ & $\mathrm{ThO}_{2}$ & 5.0 & 5.4 & +8 \\
\hline \multirow[t]{2}{*}{$\mathrm{Ti}$ in $\mathrm{HF}$ and $\mathrm{HNO}_{3}$} & $\mathrm{TiC}$ & 5.0 & 5.4 & +8 \\
\hline & $\mathrm{TiO}_{2}$ & 5.0 & 5.2 & +4 \\
\hline \multirow[t]{2}{*}{$\mathrm{Nb}$ in $\mathrm{HF}$ and $\mathrm{HNO}_{3}$} & $\mathrm{Nb}$ & 4.6 & 4. 5 & -2 \\
\hline & $\mathrm{NbC}$ & 4. 8 & 4.0 & $a_{-17}$ \\
\hline $\mathrm{Hf}$ in $\mathrm{HCl}$ and $\mathrm{HNO}_{3}$ & $\mathrm{HfC}$ & 4.7 & 4.5 & -4 \\
\hline
\end{tabular}

${ }^{a}$ Amount determined was significantly lower than amount added to anode. 
TABLE II. - EXAMPLE ANALYSIS

SHOWING MINIMAL EFFECTS

OF SAMPLE CHEMISTRY ${ }^{a}$

\begin{tabular}{|l|c|c|c|}
\hline \multicolumn{1}{|c|}{ Element } & $\begin{array}{c}\text { Standard } \\
\text { value, } \\
\text { percent }\end{array}$ & $\begin{array}{c}\text { Determined } \\
\text { value, } \\
\text { percent }\end{array}$ & $\begin{array}{c}\text { Relative } \\
\text { difference, } \\
\text { percent }\end{array}$ \\
\hline Chromium & 25.3 & 26 & +2.8 \\
Aluminum & 11.0 & 11.6 & +5.2 \\
Iron & 11.2 & 12.8 & +14 \\
Titanium & .56 & .59 & +5.4 \\
Manganese & .16 & .14 & -12 \\
\hline
\end{tabular}

${ }^{a}$ Ref. 1 describes the conditions for analysis using a $\mathrm{AgCl}$-argon arc. The sample was NBS-103, a chrome refractory ore in water slurry. The calibration standards were acid solutions of metals. The spectrometric analysis is in agreement within experimental error with the standard percentages.

TABLE III. - DISSOCIATION

ENERGY RANKING OF

MOST STABLE

METAL MONOXIDE

[Data from ref. 12.]

\begin{tabular}{|c|c|c|} 
Monoxide & \multicolumn{2}{|c|}{ Dissociation energy } \\
\cline { 2 - 3 } & $\mathrm{kJ} / \mathrm{mole}$ & $\mathrm{kcal} / \mathrm{mole}$ \\
ThO & 828 & 198 \\
TaO & 812 & 194 \\
$\mathrm{BO}$ & 799 & 191 \\
$\mathrm{SiO}$ & 795 & 190 \\
LaO & 795 & 190 \\
$\mathrm{CeO}$ & 791 & 189 \\
$\mathrm{HfO}$ & 766 & 183 \\
ZrO & 757 & 181 \\
NbO & 753 & 180 \\
\hline
\end{tabular}




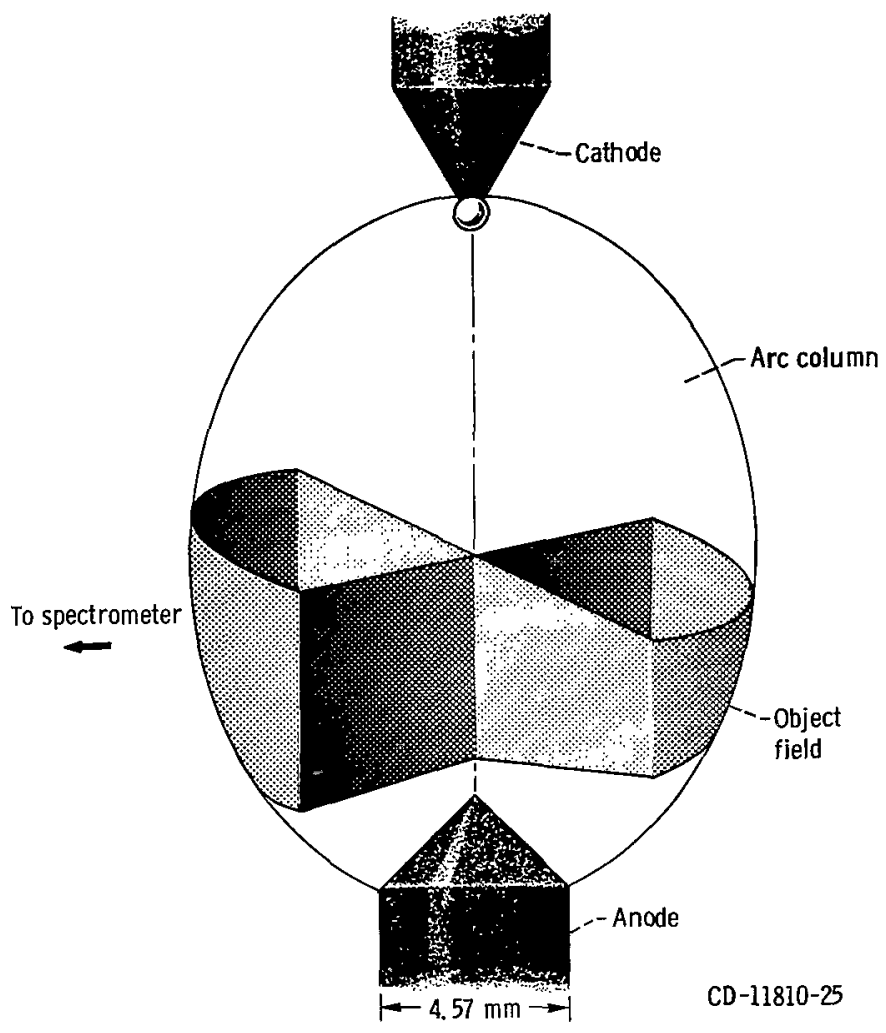

Figure 1. - Arc space configuration showing object field. Spectrometer receives emission only from within the optical volume of the object field.

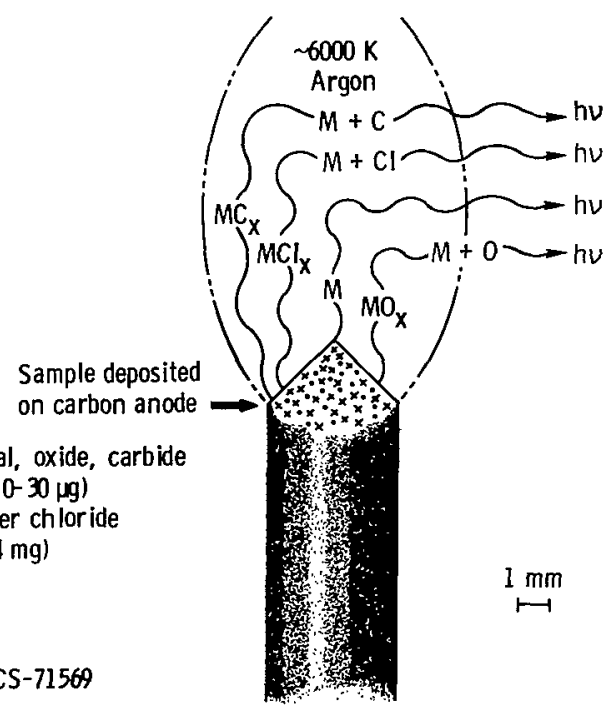

Figure 2. - Schematic of experimental conditions and fundamental processes in AgCl-argon arc. 


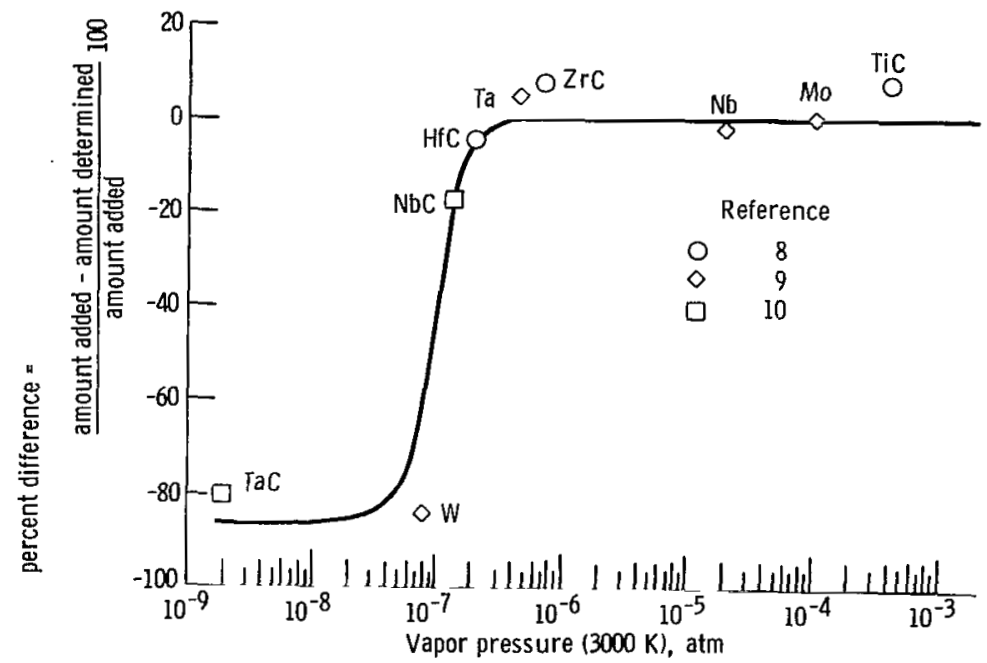

Figure 3. - Relation between vapor pressures of materials added to anodes and differences between amounts added and determined. Other compounds vaporized with differences equal to or less than \pm 10 percent and whose vapor pressures are greater than $\mathrm{IO}^{-3}$ atmosphere at $3000 \mathrm{~K}: \mathrm{Al}_{2} \mathrm{O}_{3}, \mathrm{Ta}_{2} \mathrm{O}_{5}, \mathrm{WO}_{3}, \mathrm{ZrO}_{2}, \mathrm{SiC}, \mathrm{SiO}_{2}$, $\mathrm{ThO}_{2}$, and $\mathrm{TiO}_{2}$. 
"The aeronautical and space activities of the United States sball be conducted so as to contribute ... to the expansion of buman knowledge of phenomena in the atmosphere and space. The Administration shall provide for the widest practicable and appropriate dissemination of information concerning its activities and the results thereof."

\section{NASA SCIENTIFIC AND TECHNICAL PUBLICATIONS}

TECHNICAL REPORTS: Scientific and technical information considered important, complete, and a lasting contribution to existing knowledge.

TECHNICAL NOTES: Information less broad in scope but nevertheless of importance as a contribution to existing knowledge.

TECHNICAL MEMORANDUMS:

Information receiving limited distribution because of preliminary data, security classification, or other reasons. Also includes conference proceedings with either limited or unlimited distribution.

CONTRACTOR REPORTS: Scientific and technical information generated under a NASA contract or grant and considered an important contribution to existing knowledge.
TECHNICAL TRANSLATIONS: Information published in a foreign language considered to merit NASA distribution in English.

SPECIAL PUBLICATIONS: Information derived from or of value to NASA activities. Publications include final reports of major projects, monographs, data compilations, handbooks, sourcebooks, and special bibliographies.

\section{TECHNOLOGY UTILIZATION}

PUBLICATIONS: Information on technology used by NASA that may be of particular interest in commercial and other non-aerospace applications. Publications include Tech Briefs, Technology Utilization Reports and Technology Surveys.

Defails on the availability of these publications may be obtained from:

SCIENTIFIC AND TECHNICAL INFORMATION OFFICE 